\title{
Nota del editor
}

La Facultad de Ingeniería de la Corporación Universitaria Minuto de Dios, UNIMINUTO, al tomar la determinación, en el año 2006, de publicar la revista INVENTUM, se fijó el propósito de que su publicación tenga periodicidad semestral e incluya temas de interés en los campos del saber relacionados con los programas de estudio que ofrece o que son tema del quehacer académico de sus diferentes unidades. Se trata de un esfuerzo conjunto de toda la Facultad de tal manera que en cada número se tratan diferentes temáticas pero además se selecciona un tema principal y se asigna una responsabilidad especial al programa o dependencia a que él corresponde.

En este número el tema principal es el de Redes de Computadores y la Seguridad Informática, que corresponde al programa tecnológico con el mismo nombre, que se comenzó a ofrecer en el año 2004; tres son los artículos que se refieren a esta temática. Hay además dos artículos relacionados con programación y manejo de información, asuntos que tienen relación con la Tecnología en Informática, programa que está graduando tecnólogos desde el primer semestre del año 2004. Los otros tres artículos que se presentan en este número están basados en trabajos de grado seleccionados por los correspondientes cuerpos profesorales, dos sobre investigaciones en el campo de ciencias agrícolas, correspondientes al programa en Ingeniería Agroecológica, y uno sobre cargas estructurales dinámicas realizado por dos graduandos de Ingeniería Civil.

En cuanto a los autores se puede comentar que dos de ellos son invitados; para los tres artículos basados en trabajos de grado se tienen las coautorias de los respectivos asesores de proyecto, dos en los de Ingeaniería Agroecológica al incluir al Dr. Everth Ebratt quien es investigador del ICA, en el Centro de Investigaciones de Tibaitatá, donde se efectuaron las investigaciones dentro de convenio vigente entre las dos instituciones; el resto de los autores son profesionales vinculados a UNIMINUTO. Los trabajos de los tesistas de Ingeniería Agroecológica fueron motivo de presentación en Congreso de Entomología realizado en Cartagena este año.

Por último se hace un reconocimiento especial a la labor realizada por el Comité Editorial de la revista, al anterior director Lic. Dairo Muñoz y la coordinadora de la publicación Ing. Libia García. 